\title{
Ion Source Optimisation for Proton Beam Quality of the Van De Graaff Accelerator at iThemba LABS for Ion Beam Analysis
}

\author{
M. E. M. Eisa ${ }^{1,2^{*}}$, J. L. Conradie ${ }^{3}$, P. J. Celliers ${ }^{3}$, J. L. G. Delsink ${ }^{3}$, D. T. Fourie ${ }^{3}$, G. de Villiers ${ }^{3}$, \\ K. A. Springhorn ${ }^{4}$, C. A. Pineda-Vargas ${ }^{4,5}$ \\ ${ }^{1}$ Department of Physics, Northern Border University, Arar, Kingdom of Saudi Arabia \\ ${ }^{2}$ Department of Physics, Sudan University of Science and Technology, Khartoum, Sudan \\ ${ }^{3}$ Accelerator Group, iThemba LABS, Somerset West, South Africa \\ ${ }^{4}$ iThemba LABS, Materials Research Department, Somerset West, South Africa \\ ${ }^{5}$ Faculty of Health and Wellness Sciences, C.P.U.T, Bellville, South Africa \\ Email: "memeisa@yahoo.com
}

Received April 18, 2013; revised May 20, 2013; accepted June 1, 2013

Copyright (C) 2013 M. E. M. Eisa et al. This is an open access article distributed under the Creative Commons Attribution License, which permits unrestricted use, distribution, and reproduction in any medium, provided the original work is properly cited.

\begin{abstract}
The operating principles of the two duoplasmatron ion sources and the PIG source available for the Van de Graaff accelerator have been determined qualitatively, mainly by studying the extensive literature on ion sources. The main characteristics of the three sources have therefore been determined experimentally on an ion source test bench. Important parameters of the sources such as the pressure and temperature in different regions of the sources not measured, because of space limitations. However, in spite of these limitations in quantitative understanding of the sources, sufficient information to continue with the study of the beam transport through the Van de Graaff accelerator and its beamlines could be obtained from these measurements. With regard to beam intensity, life-time and gas consumption the measurements showed that the hot-cathode duoplasmatron is at present by far the most suitable source for the van de Graaff accelerator. The emittance of the ion source was measured with two slits, $90 \mathrm{~mm}$ apart, and a Faraday cup in the ion source test bench at an arc voltage of $83 \mathrm{~V}$, an arc current of $1 \mathrm{Amp}$, a filament voltage of $24 \mathrm{~V}$ and an extraction voltage of $5 \mathrm{kV}$. The measured emittance for $90 \%$ of the beam intensity is $48 \pi \mathrm{mm}$ mrad. This figure will be used to calculate the beam transmission through the Van de Graaf accelerator. Different computer programs have been used for these calculations from the ion source through the terminal section, the accelerator and beam line up to the Nuclear Microprobe (NMP) for beam quality.
\end{abstract}

Keywords: Van De Graaff; Beam Optics; Ion Source

\section{Introduction}

At iThemba LABS two duoplasmatron ion sources with hot and cold cathodes, respectively, as well as a coldcathode Penning Ionization Gauge (PIG) source are available for the Van de Graaff accelerator. However, this study will report only on the hot cathode duoplasmatron since it is more commonly used for IBA experiments. The optimum operating conditions, such as the filament current, cathode and anode voltages and the gas flow under which stable beams can be delivered reliably to the users with an acceptable long source lifetime, were determined, since no experimental data were available[1].

${ }^{*}$ Corresponding author.
The beam quality and intensity have not been sufficient for high-resolution Ion Beam Analysis (IBA) and in particular Nuclear Microprobe (NMP) work. Previous studies have shown that ion source stability and efficiency is the most important factor to ensure a reliable beam probe at the NMP target surface. Major efforts have been made recently to improve the reliability of this accelerator [2,3]. However computer programs such as TRANSPORT program [4,5] which calculates first-, second- and thirdorder effects by matrix multiplication. Matrices are used to describe the beam optical elements and the charged particle beam itself. The program can also vary the physical parameters of the line to fit the elements of matrices in order to obtain values and conditions, which are speci- 
fied by the user, and TOSCA [6] is analysis package, for the calculation of three dimensional magnetostatic and electrostatic fields, a module of the OPERA-3d integrated suite of finite element software for 3D electromagnetic design, analysis and simulation, is a commercial software product from Vector Fields. The TOSCAmodule uses a discrete finite element model to solve partial differential equations governing the behavior of static electric and magnetic fields in three dimensions. In order to include space-charge effects in the calculations the program IGUN [7-9] which is a two-dimensional ray tracing code that has been developed for simulation of the extraction of positive ions from plasmas. A preprocessor, GPED (an acronym for Graphic Polygon Editor) for IGUN, has also been developed to set up the boundary conditions, including the definition of internal electrodes, dielectric boundaries, and slanted Neumann boundaries (field lines as boundary elements). The results obtained with the different three codes were discussed and agreed with each other and also compared with experimentally obtained data. Finally, the applicability of the three computer codes for this specific task is reviewed.

\section{Measurements and Results}

Since it is not possible to calculate the performance of ion sources with accuracy of the dimensions of the electrodes, the gas flow and the applied voltages and currents, it is standard practice to determine the source characteristics on a test bench [10]. Figure 1 shows a layout of the ion source test bench at iThemba LABS. The ion source under test and all its power supplies are at a positive potential, the extraction voltage, with respect to the beam pipe on the test bench. The diagnostic equipment consists of two Faraday cups for the measurement of the beam intensity and adjustable horizontal and vertical slits for selection of portions of the beam, after it has passed through the pole gap of a bending magnet and a drift space. The vertical slit has two independently adjustable jaws to select ions with a specific charge-to-mass ratio within a limited momentum range. For a $1 \mathrm{~mm}$ object slit width, i.e. the aperture in the extraction electrode directly in front of the ion source, the charge state, the mass and momentum resolving power at the vertical slit jaws is $0.9 \%$. For the same slit size the energy resolving power is $1.8 \%$. Downstream from the vertical slit two horizontal slits with fixed apertures of $1 \mathrm{~mm}$, but with adjustable positions, are installed at a distance of $90 \mathrm{~mm}$ apart for emittance measurement [11].

\subsection{Optimization of the Ion Source by Varying the Gas Flow}

The gas flow to the ion source was varied to determine

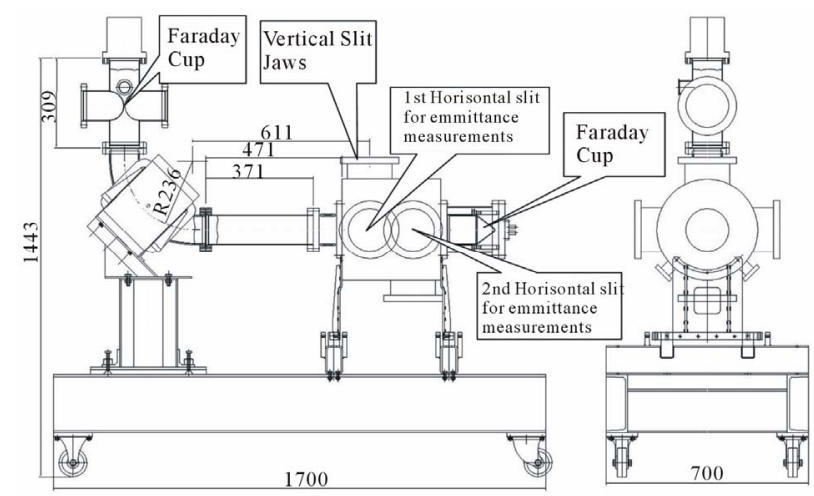

Figure 1. Layout of the ion source test bench at iThemba LABS showing side (left) and front (right) views. The dimensions in the figure are in millimeters.

the optimum condition in terms of intensity for protons. The gas consumption of the duoplasmatron ion source is, however, so low that that the gas flow could not be measured with the available equipment. Because of the limitations of size the pressure could also not be measured in the ion source on the test bench. Below a pressure of $6 \times 10^{-5}$ mbar the ion source becomes unstable. The maximum current for protons is obtained at a pressure slightly above the gas flow at which instability sets in. This fact can be used to adjust the gas flow when the source is used in the Van de Graaff accelerator. At pressure values below $9 \times 10^{-6}$ mbar the proton beam current increases sharply with decreasing pressure. The ion source is at a positive voltage of several kilovolts with respect to the extractor electrode. The total beam current as a function of the arc current at an extraction voltage of $11 \mathrm{kV}$. At the Van de Graaff the maximum extraction voltage is, however, limited to $8 \mathrm{kV}$ to prevent excessive sparking. At this voltage a beam current of only $6 \mu \mathrm{A}$ is obtained for protons. The source can be operated stably up to arc currents of 50 to $60 \mathrm{~mA}$. Above that the source becomes unstable due to excessive heating of the electrodes. This source is therefore on account of its low beam current of little use for the Van de Graaff accelerator. This shows that to obtain intense proton beams the source should be operated at a pressure just above the value at which the source becomes unstable. Emittance measurements, similar to those of the hot-cathode duoplasmatron ion source have been made for the PIG ion source. A value of $34 \pi \mathrm{mm}$ mrad for $96 \%$ of the beam intensity has been obtained.

\subsection{Optimization of the Ion Source by Varying the Extraction Voltage for Both the PIG and Duoplasmatron Ion Sources}

The solid lines in Figure 2 shows the measured beam current for different Hydrogen ion species as a function of the extraction voltage. The remaining ion source pa- 


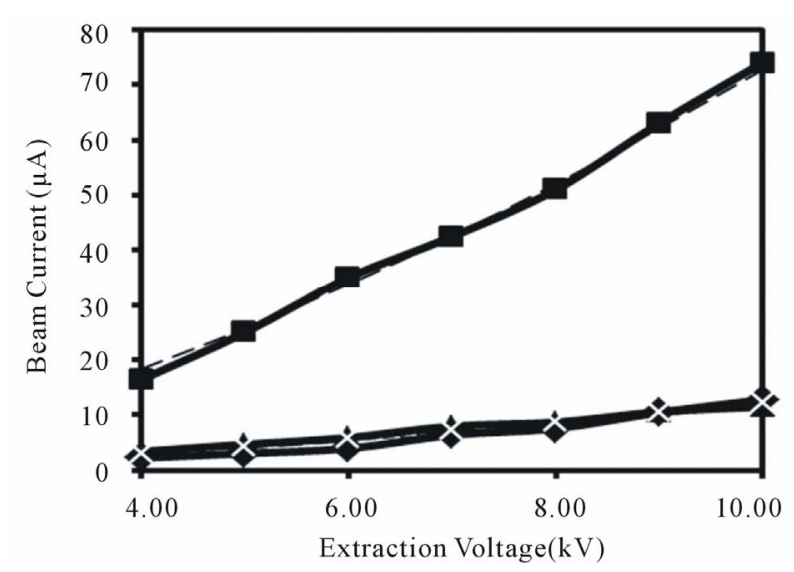

Figure 2. Measured beam current (solid lines) for different Hydrogen ion species as a function of the extraction voltage, for the Penning ion source and leasts quare fits (dotted lines) of the Child-Langmuir law. The measurements were made under the following conditions: arc current $=1 \mathrm{~A}$, arc voltage $=800 \mathrm{~V}$, pressure $=4 \times 10^{-5} \mathrm{mBar}$, magnet power supply $=\mathbf{5 0} \mathrm{V}$.

rameters were kept at the following constant values: arc current $=1 \mathrm{~A}$, arc voltage $=800 \mathrm{~V}$, pressure $=4 \times 10^{-5}$ mbar and $50 \mathrm{~V}$ across the magnet coil. As before for the hot-cathode duoplasmatron source it is expected that the beam current as a function of the extraction voltage should follow the Child-Langmuir law, i.e. the extracted ion beam current I should be proportional to the extraction voltage $\mathrm{V}^{3 / 2}$. The dotted lines in Figure 3 are least-square fits of the equation $\mathrm{I}=$ constant $\times \mathrm{V}^{3 / 2}$ to the measured data for the different ion species and show that the source behavior is to a large extent in agreement with the Child-Langmuir law which means that the beam current extracted from the source is limited by the space charge in the region between the anode and extraction electrode. It again also indicates that the beam losses in the beam line due to space-charge effects are small and only of importance for operation with low extraction voltages, as shown in Figure 3. For high beam intensities the source should be function of the positions of the two slits is shown in Figure 4.

\section{Conclusion and Discussion}

The operating principles of the two duoplasmatron ion sources and the PIG source available for the Van de Graaff accelerator have been determined qualitatively, mainly by studying the extensive literature on ion sources. No quantitative treatment of such sources by which their performance can be calculated in terms of their dimensions, the applied voltages and the gas flow could be obtained from the literature. The main characteristics of the three sources have therefore been determined experimentally on an ion source test bench. Important parameters of the sources such as the pressure

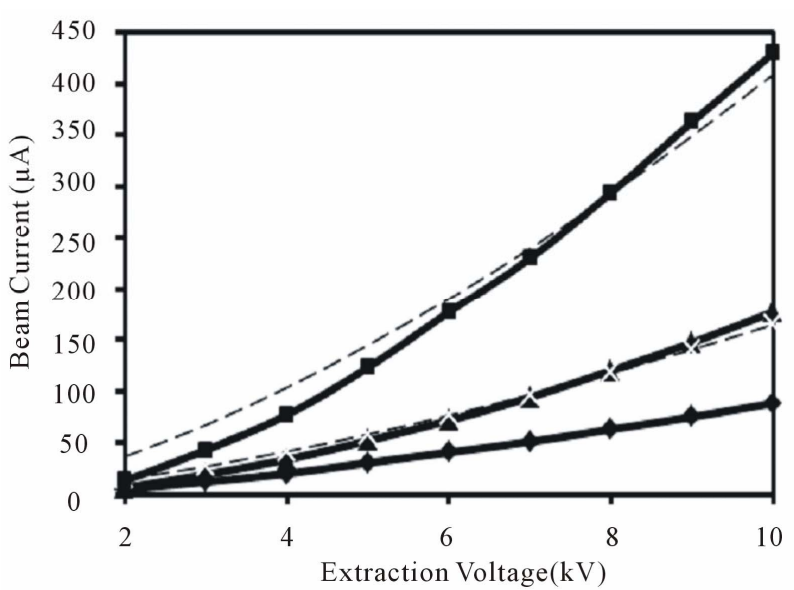

Figure 3. Measured beam current (solid lines) for different Hydrogen ion species as a function of the extraction voltage, for the hot-cathode duoplasmatron ion source and leastsquare fits (dotted lines) of the Child-Langmuir law. The measurements were made under the following conditions: arc current $=1 \mathrm{~A}$, arc voltage $=83 \mathrm{~V}$, filament.

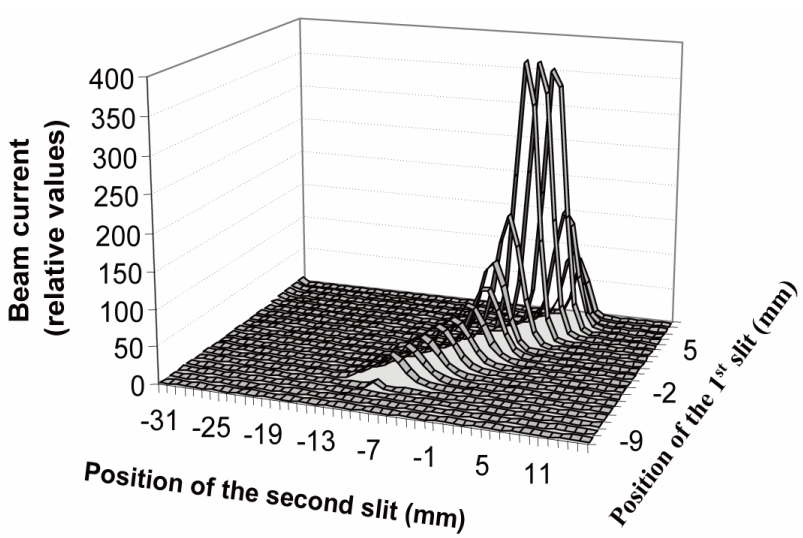

Figure 4. The beam intensity distribution for the hot- cathode duoplasmatron ion source at different positions of the two slits in the ion source test bench with which the emittance was measured.

and temperature in different regions of the sources could, because of space limitations, not be measured. With regard to beam intensity, life-time and gas consumption the measurements showed that the hot-cathode duoplasmatron is at present by far the most suitable source for the van de Graaff accelerator. In future microwave ion sources could be considered for the Van de Graaff accelerator for higher beam intensities and better beam quality, the problems experienced in the past with poor beam transmission below terminal voltages of $3 \mathrm{MV}$ and the limitation of the maximum extraction voltage to $5 \mathrm{kV}$ could be explained. A simple and complete solution to these problems was found by using existing electrodes in the terminal section, and without modifying this very complex structure, as an additional einzel lens. The measured emittance for $90 \%$ of the beam intensity was $48 \pi$ 
$\mathrm{mm} \mathrm{mrad}$. This result was used for the calculation of the beam transmission through the Van de Graaf accelerator and beam line up to the Nuclear Microprobe (NMP) for beam quality and intensity. Beam reliability at the NMP instrument has resulted in an improvement of the probe resolution at the target focal plane $(\sim 1-3 \mu \mathrm{m}$ in size), for protons energies between 1 and $4 \mathrm{MeV}$. The new probe stability has allowed better microprobe work especially in the mapping mode with current stability acceptable for long periods of time. Some of the results obtained in applications to bio-medical samples $[12,13]$.

\section{Acknowledgements}

The authors would like to thank the staff of the Accelerator and Materials Research Groups at iThemba LABS for their support throughout the experiments and measurements. One of the authors M. E. M. Eisa is thanking Sudan University of Science \& Technology for support and TWAS-UNESCO for their financial support during the completion of this investigation.

\section{REFERENCES}

[1] J. L. Conradie, M. E. M. Eisa, P. J. Celliers, J. L. G. Delsink, D. T. Fourie, J. G. de Villiers, P. M. Maine, K. A. Springhorn and C. A. Pineda-Vargas, "Recent Optimization of the Beam-Optical Characteristics of the $6 \mathrm{MV}$ van de Graaff Accelerator for High Brightness Beams at the iThemba LABS NMP Facility," Nuclear Instruments and Methods in Physics Research Section B: Beam Interactions with Materials and Atoms, Vol. 231, No. 1-4, 2005, pp. 101-105. doi:10.1016/j.nimb.2005.01.041

[2] G. W. Grime and F. Watt, "Focusing Protons and Light ions to Micron and Submicron Dimensions," Nuclear Instruments and Methods in Physics Research Section B: Beam Interactions with Materials and Atoms, Vol. 30, No. 3,1988 , pp. 227-234. doi:10.1016/0168-583X(88)90002-X

[3] S. Kalbitzer, "Bright Ion Beams for the Nuclear Microprobe," Nuclear Instruments and Methods in Physics Research Section B: Beam Interactions with Materials and Atoms, Vol. 158, No. 1-3, 1999, pp. 53-60. doi:10.1016/S0168-583X(99)00324-9
[4] D. C. Carey, "Computer Simulation of Charged Particle beams," Nuclear Instruments and Methods, Vol. 104, No. 1, 1972, pp. 173-178. doi:10.1016/0029-554X(72)90314-X

[5] D. C. Carey, "High Energy Charged Particle Optics Computer Programs," Nuclear Instruments and Methods in Physics Research, Vol. 187, No. 1, 1981, pp. 97-102. doi:10.1016/0029-554X(81)90475-4

[6] J. Borburgh, "2-Dimensional Finite Element Calculations on a Septum Magnet with Opera2D," CERN Note PS/CA/Note 97-27.

[7] R. Becker and W. B. Herrmannsfeldt, "IGUN-A Program for the Simulation of Positive Ion Extraction Including Magnetic Fields," Review of Scientific Instruments, Vol. 63, No. 4, 1992, pp. 2756-2761.

doi:10.1063/1.1142795

[8] R. Becker, "INTMAG: A Program for the Calculation of Magnetic Fields by Integration," Nuclear Instruments and Methods in Physics Research Section B: Beam Interactions with Materials and Atoms, Vol. 42, No. 3, 1993, pp. 303-306.

[9] R. Becker, "New Features in the Simulation of Ion Extraction with IGUN," Proceedings of the 6th European Particle Accelerator Conference, Stockholm, June 1998, p. 1165.

[10] M. L. Mallory and D. H. Crandall, "A Penning Multiply Charged Heavy Ion Source Test Facility," IEEE Transactions on Nuclear Science, Vol. 23, No. 2, 1976, pp. 10691072. doi:10.1109/TNS.1976.4328405

[11] M. E. M. Eisa, "Optimization of Accelerator Optics and the Application of Nuclear Microscopy to the Biomedical Field," Ph.D. Dissertation, University of Cape Town, 2005.

[12] C. A. Pineda-Vargas, M. Topic, T. Ntsoane, W. J. Przybylowicz and M. Harting, "Nuclear Microprobe Analyses of Bioconductive Hydroxyapatite Coatings," International Conference on Nuclear Microprobe Technology and Applications, Cavtat, 13-17 September 2004.

[13] C. A. Pineda-Vargas, M. E. Eisa, "Analysis of Human Hair Cross Sections from Two Different Population Groups by Nuclear Microscopy," Nuclear Instruments and Methods in Physics Research Section B: Beam Interactions with Materials and Atoms, Vol. 268, No. 11-12, 2010, pp. 2164-2167. doi:10.1016/j.nimb.2010.02.041 